\title{
A randomised controlled trial of azithromycin to prevent chronic rejection after lung transplantation
}

\author{
R. Vos*,", B.M. Vanaudenaerde*,", S.E. Verleden*,\#, S.I. De Vleeschauwer*\#, \\ A. Willems-Widyastuti*,*, D.E. Van Raemdonck ${ }^{\#, \uparrow}$, A. Schoonis ${ }^{\#}$, T.S. Nawrot ${ }^{+, \S}$, \\ L.J. Dupont ${ }^{\#}$ and G.M. Verleden ${ }^{\#}$
}

ABSTRACT: Azithromycin reduces airway inflammation and improves forced expiratory volume in $1 \mathrm{~s}$ (FEV 1 ) in chronic rejection or bronchiolitis obliterans syndrome (BOS) after lung transplantation (LTX). Azithromycin prophylaxis might prevent BOS.

A double-blind randomised controlled trial of azithromycin $(n=40)$ or placebo $(n=43)$, initiated at discharge and administered three times a week for $2 \mathrm{yrs,} \mathrm{was} \mathrm{performed} \mathrm{in} \mathrm{2005-2009} \mathrm{at} \mathrm{the}$ Leuven University Hospital (Leuven, Belgium). Primary end-points were BOS-free and overall survival 2 yrs after LTx; secondary end-points were acute rejection, lymphocytic bronchiolitis and pneumonitis rate, prevalence of pseudomonal airway colonisation or gastro-oesophageal reflux, and change in FEV 1 , airway and systemic inflammation over time. Patients developing BOS were assessed for change in FEV 1 with open-label azithromycin.

BOS occurred less in patients receiving azithromycin: 12.5 versus $44.2 \%(p=0.0017)$. BOS-free survival was better with azithromycin (hazard ratio $0.27,95 \% \mathrm{Cl} 0.092-0.816 ; p=0.020$ ). Overall survival, acute rejection, lymphocytic bronchiolitis, pneumonitis, colonisation and reflux were comparable between groups. Patients receiving azithromycin demonstrated better FEV 1 $(p=0.028)$, and lower airway neutrophilia $(p=0.015)$ and systemic C-reactive protein levels $(p=0.050)$ over time. Open-label azithromycin for BOS improved $\mathrm{FEV}_{1}$ in $\mathbf{5 2 . 2 \%}$ patients. No serious adverse events were noted.

Azithromycin prophylaxis attenuates local and systemic inflammation, improves FEV 1 and reduces BOS 2 yrs after LTX.

KEYWORDS: Azithromycin, bronchiolitis obliterans syndrome, lung allograft rejection, lung transplantation, obliterative bronchiolitis

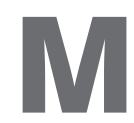
ean actuarial 5-yr survival rate after lung transplantation is currently $\sim 50 \%$, increasing to $70 \%$ in some high-volume centres $[1,2]$. This is far behind other solid organ transplantations $[3,4]$. Death most often results from chronic rejection [1], which presents histologically as obliterative bronchiolitis, a scarring of the terminal bronchioles [5-8], and clinically as obstruction identified by spirometry, defined as bronchiolitis obliterans syndrome (BOS), which has a 5 -yr prevalence of $45 \%[1,8,9]$. Because obliterative bronchiolitis is not consistently detectable by transbronchial biopsy, spirometry is routinely used as a surrogate marker to diagnose chronic rejection. BOS is thought to be the final common end-point of various alloimmunologic and nonalloimmunologic injuries to the pulmonary allograft [10, 11]. Preventive and therapeutic strategies for BOS have been largely unsuccessful [11-15]. However, since the recent introduction of the neomacrolide antibiotic azithromycin in the field of lung transplantation, it has become clear that, currently, some $35 \%$ of patients with established BOS can be adequately treated, in particular those with an increased bronchoalveolar lavage neutrophilia of $15-20 \%$ or more [16-24]. In these patients, azithromycin treatment resulted in a mean increase in forced expiratory volume in $1 \mathrm{~s}$ (FEV1) of $\sim 15 \%$ and possibly an improved survival [16-25].

For editorial comments see page 10.

This article has supplementary material available from www.erj.ersjournals.com 
In view of the significant morbidity and mortality associated with BOS, as well as the enormous healthcare costs attributed to frequent hospitalisations, extensive immunosuppressive treatment and use of diagnostic procedures in these patients [26], we investigated whether prophylactic azithromycin treatment would improve outcome (i.e. BOS-free and overall survival) after lung transplantation.

\section{METHODS}

\section{Study design}

A randomised, double-blind, placebo-controlled trial of oral azithromycin, given in addition to conventional immunosuppression, was conducted at the Leuven University Hospital (Leuven, Belgium) with the approval of the institutional review board. Single-lung, bilateral lung or heart-lung transplant recipients who were $\geqslant 18$ yrs of age were eligible. Patients were excluded if they had an intensive care unit stay of $>30$ days or had died $<30$ days post-transplantation, had an important bronchial stenosis, underwent retransplantation or multiorgan transplantation (lung plus a solid organ) or had undergone solid-organ or bone marrow transplantation in the past. In September 2005-December 2007, patients were offered enrolment if they met the study criteria at discharge after transplantation. Immediately after the provision of written informed consent, patients were randomly assigned to groups through permuted block randomisation in a 1:1 ratio by the Hospital Experimental Pharmacy (Leuven University Hospital) to receive either oral azithromycin or placebo for 2 yrs. Study treatment was initiated as soon as possible thereafter, but no more than 30 days later. Participants, nurses administering the study medication and principal investigators (L.J. Dupont and G.M. Verleden; both Katholieke Universiteit Leuven and University Hospital Gasthuisberg, Leuven, Belgium) were blinded to group assignment throughout the entire study period. All evaluations of outcomes were performed in a blinded manner by University investigators from the Laboratory of Pneumology (R. Vos and B.M. Vanaudenaerde; both Katholieke Universiteit Leuven and University Hospital Gasthuisberg). University investigators were solely responsible for the trial design, data collection, study management and data analyses presented in this article. The data and safety monitoring board (Clinical Trial Center, UZ Leuven, Leuven, Belgium) took no action regarding early trial cessation.

\section{Administration of investigational medicinal product}

Azithromycin (Zithromax ${ }^{\mathrm{TM}}$, azithromycin dihydrate, $250 \mathrm{mg}$ hard gelatine capsule formulation) was purchased from Pfizer (Sandwich, UK) and overencapsulated by the University's Hospital Experimental Pharmacy. For placebo, lactose monohydricum Ph. Eur. was purchased from Fagron (Rotterdam, the Netherlands) and compounded into $300 \mathrm{mg}$ hard gelatine capsules by the University's Hospital Experimental Pharmacy. Study medication was provided in numbered containers to the patients during routine follow-up visits at the outpatient clinic or during hospital admissions by a study nurse who also verified compliance and possible adverse events at each contact. The study drug was initiated at one dose a day for five consecutive days, followed by one dose three times a week on Mondays, Wednesdays and Fridays. Patients were instructed to continue treatment for 2 yrs. If a patient developed BOS, the study drug was stopped (without deblinding of the investigational drug) and the patient was initiated on open-label "rescue" treatment of azithromycin for ethical reasons, initiated at $250 \mathrm{mg}$ daily for 5 days, followed by $250 \mathrm{mg}$ three times a week.

\section{Transplant monitoring and clinical management}

Patients followed a typical, standardised outpatient care regimen after lung transplantation, with complete history and physical examination, blood work, urine, sputum and pharyngeal swab cultures, spirometry and chest radiography at twice-weekly intervals for the first two post-operative months, at weekly to biweekly intervals thereafter until 6 months post-transplantation, then every 2-4 weeks until the first post-operative year and, afterwards, life-long at intervals of 2-3 months. Surveillance bronchoscopy and bronchoalveolar lavage was routinely performed at specific time-points after transplantation (at discharge, and 3, 6, 12, 18 and 24 months post-transplantation, and later at intervals of $1 \mathrm{yr}$ ) in case of clinically suspected acute allograft rejection, infection or BOS. Transbronchial biopsies were routinely performed at discharge and 3 months post-transplantation in case of suspected acute rejection, infection or BOS. Bronchoscopic procedures and processing of specimens are comprehensively described in the supplementary material. Histologic rejection was defined according to established criteria [7]. The presence of obliterative bronchiolitis was assessed at autopsy or on explant biopsies in case of retransplantation. Spirometry (Masterscreen; Jaeger, Hoechberg, Germany) was performed according to American Thoracic Society standards [27] and FEV1 \% predicted [28]. BOS was defined as a sustained decrease in FEV1 of $\geqslant 20 \%$ from the patient's maximum postoperative values in the absence of other causes [9]. For patients initiated on open-label "rescue" treatment, response to azithromycin was defined as an increase in FEV1 of $\geqslant 10 \%$ after 3-6 months of treatment and nonresponders were defined as having an increase of $<10 \%$ or a further decrease [21]. Survival data were obtained using mortality information in the Leuven University Hospital transplant database, in which all our lung transplant recipients since $1991(n=510$ on January 1, 2010) are registered.

\section{Therapeutic management}

After discharge, both groups received conventional triple-drug immunosuppression with methylprednisolone, a calcineurin inhibitor (cyclosporine A or tacrolimus) and a cytostatic agent (azathioprine or mycophenolate mofetil), as comprehensively described in the supplementary material. Drug choice and dosing adjustments were made according to a standardised protocol at the discretion of the treating clinician on the basis of renal function, immunosuppressive trough levels, spirometry and biopsy results. Patients developing BOS were treated with azithromycin according to the open-label trial protocol and in the case of BOS progression with conversion of immunosuppressives or, less frequently, total lymph node irradiation or retransplantation.

All patients received conventional infectious prophylaxis for cytomegalovirus (CMV), Aspergillus spp. and Pneumocystis spp., as described in the supplementary material. CMV-related disease or pneumonitis was treated with Ganciclovir. 
Antibiotic treatment for bacterial infection was guided using bacterial cultures.

All patients received conventional prophylaxis for gastrooesophageal reflux [29] and confirmed reflux was treated by a high-dose proton pump inhibitor, as described in the supplementary material. When the current study was initiated, no standardised protocol for the evaluation of reflux had been instituted at our centre; therefore, reflux was assessed either by $\mathrm{pH}$ impedance measurement or by gastroscopy at variable time-points during the first 2 yrs after transplantation.

\section{End-points}

Primary end-points were the prevalence of BOS and overall survival at 2 yrs after transplantation. Secondary end-points included the rate of histological acute rejection, lymphocytic bronchiolitis and pulmonary infection, and the prevalence of pseudomonal airway colonisation and gastro-oesophageal reflux, as well as change in FEV1, and airway and systemic inflammation over time during the study.

Adverse events were defined as serious allergic reactions including skin reactions (rash, urticaria or Stevens-Johnson syndrome), angioneurotic oedema and anaphylaxis, cardiac arrhythmias (ventricular tachycardia or Torsades de Pointes), neurologic disorders (convulsions), gastrointestinal disorders including abdominal discomfort (nausea, dyspepsia, pain or cramps), diarrhoea or pseudomembranous colitis.

\section{Statistical analysis}

Power analysis stipulating a 30\% difference in the prevalence of BOS after 2 yrs suggested enrolment of $\geqslant 82$ patients based on a two-sided test $(\alpha=0.05, \beta=0.20)$, a BOS prevalence of $30 \%$ 2 yrs after transplantation and a dropout rate of $10 \%$. The anticipated enrolment period was 3 yrs, based on a mean annual number of 39 transplantations in the preceding $3.5 \mathrm{yrs}$ and an exclusion rate of $30 \%$. Actual enrolment of 83 eligible patients could already be discontinued after 2.4 yrs due to a higher number of transplantations performed during this data collection period (mean annual number of 49 transplantations). Analyses were performed 2 yrs after the last subject had been enrolled, without unblinding of the randomisation to either the treating physicians or the enrolled patients and the study protocol specified a second analysis 3 yrs after enrolment of the last patient. All patients were in life-long follow-up and outcome variables were followed until the patient's death, independent of the continuation or discontinuation of study medication or the conclusion of the scheduled 2-yr study treatment period. Included patients were analysed according to the intention-to-treat principle and no patient was lost to follow-up.

Group means were compared using unpaired, two-tailed ttests or Mann-Whitney tests for non-normally and normally distributed variables, respectively (Graphpad Prism 4.0 software, San Diego, CA, USA). The Chi-squared test was used to compare proportions. All reported p-values are two-sided and have not been adjusted for multiple testing. Kaplan-Meier estimates with log-rank tests were used for time-to-event analyses. For the end-point of BOS, survival times were censored at death, retransplantation or at study discontinuation if these preceded BOS, or else at 2 yrs after transplantation. For the end-point of death, survival times were not censored at retransplantation or at study discontinuation if these preceded death, or else at 2 yrs after transplantation. Additional Cox proportional-hazards analyses (SAS 9.1 Institute Inc. Software, Cary, NC, USA) were used to compare BOS-free survival and overall survival between groups with or without adjustment for type of transplantation (single or bilateral lung), recipient and donor age, recipient sex, recipient CMV mismatch status (positive donor to negative recipient), the rate of acute rejection (histological grade A), lymphocytic bronchiolitis (histological grade $\mathrm{B}$ ), and $\mathrm{CMV}$ and non-CMV pneumonitis. The frequency or rate of specific events (i.e. acute rejection, lymphocytic bronchiolitis, CMV and non-CMV pneumonitis) was calculated by determining the number of events per yr of study time for each subject. Mixed-effect models were used to test longitudinal effects on repeated measurements of FEV1, bronchoalveolar lavage neutrophilia and plasma C-reactive protein. Because within-individual repeated measures of outcomes are correlated, random effects were estimated at the subject level, nested within therapy group. To achieve normal distributions of neutrophilia and C-reactive protein, we applied Box-Cox transformations. Q-Q plots of the residuals, the residuals versus the fitted values and the residuals versus leverage were used to test the assumptions of all linear models.

\section{RESULTS}

\section{Patients' characteristics}

Of the 119 patients transplanted during the enrolment period, 83 were randomly assigned to a study group (placebo $n=43$; azithromycin $n=40$ ) (fig. 1). Baseline characteristics were similar in both groups (table 1), as was immunosuppressive management (supplementary table S1). The number of spirometric measurements, bronchoscopic and transbronchial biopsy procedures were similar between both groups (supplementary table S2). The mean \pm SD duration of treatment was $498.2 \pm 224.7$ days among patients receiving placebo and $565.7 \pm 224.5$ days among patients receiving azithromycin $(\mathrm{p}=0.17) .18(41.9 \%)$ out of the 43 patients in the placebo group and $28(70.0 \%)$ of the 40 patients in the azithromycin group completed the 2-yr study drug treatment period. Reasons for discontinuation are given in table 2. Overall, there were no significant differences between groups regarding study drug discontinuation. Discontinuation was initiated either by the patients (i.e. patients tolerated the study drug but withdrew from the study) or by the investigators (mainly because of concerns regarding gastro-intestinal intolerance with diarrhoea), which accounted for most of the discontinuations in the placebo group $(4.6 \%)$ and the azithromycin group $(5.0 \%)$, respectively. One patient in each group was withdrawn from the study because of the development of pulmonary malignancy requiring systemic chemotherapy or unilateral pneumonectomy. Two patients in the azithromycin group were withdrawn either because of severe sepsis due to a liver abscess or late onset suture stenosis due to local Aspergillus infection, leading to the patient's death in the former case and obscuring further spirometric assessments in the latter. 18 $(41.9 \%)$ patients from the placebo group and five (12.5\%) from the azithromycin group were initiated on open-label azithromycin treatment after having developed BOS. One patient from the placebo group could not be assessed for subsequent change in FEV1 over time due to an episode of severe 


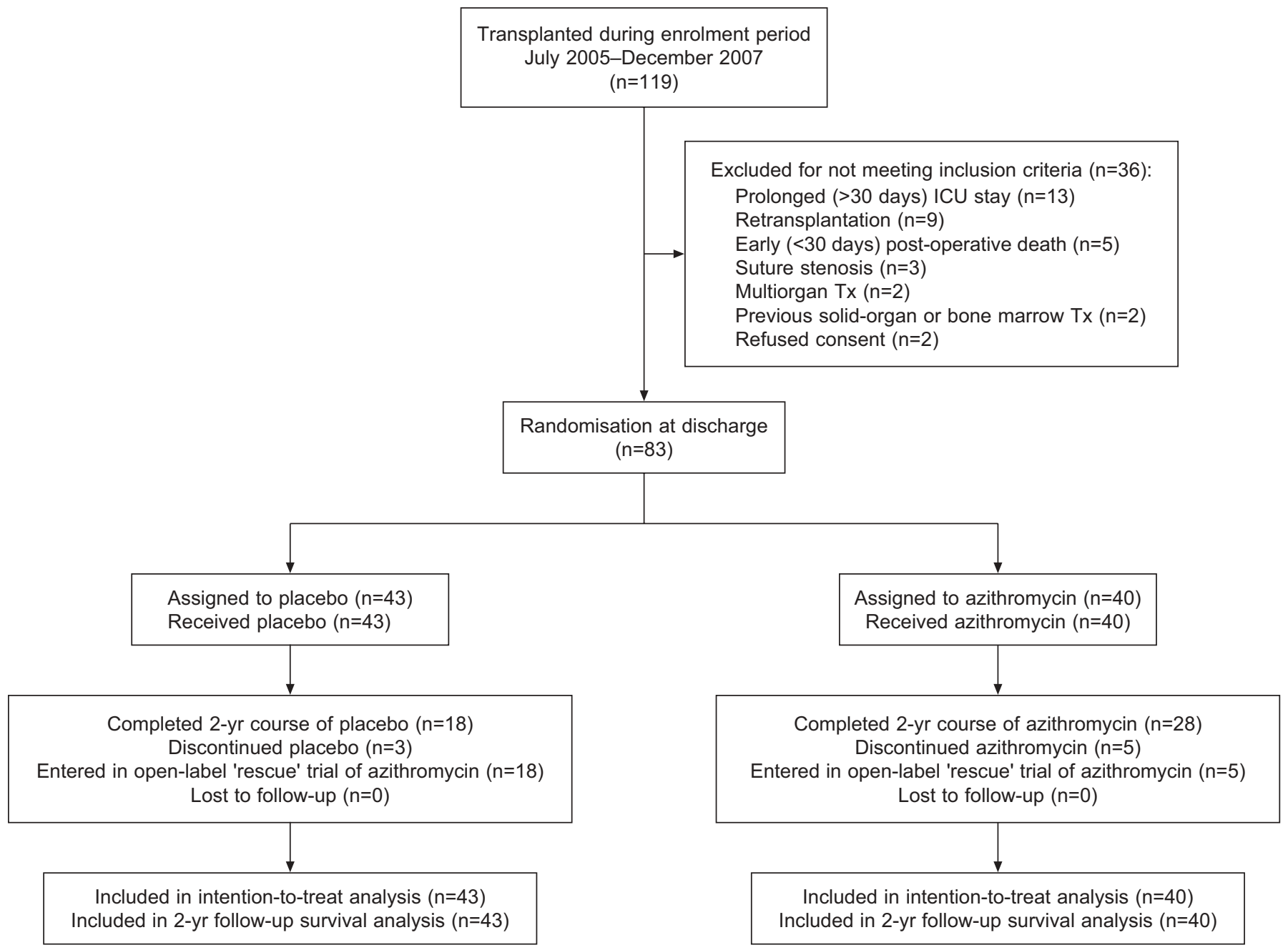

FIGURE 1. Study enrolment and inclusion in the intention-to-treat and survival analyses. ICU: intensive care unit; Tx: transplant.

mucormycosis-sepsis and death $\leqslant 30$ days after initiation of open-label treatment. Data regarding changes in FEV1 after the initiation of open-label treatment were separately analysed and not included in the initial intention-to-treat analysis.

\section{Primary end-points}

Chronic rejection

Chronic rejection occurred significantly less in patients receiving azithromycin compared with patients receiving placebo: five $(12.5 \%)$ versus $19(44.2 \%)$ events at 2 yrs after transplantation, respectively $(\mathrm{p}=0.0017)$. BOS-free survival was improved among patients treated with azithromycin compared to placebo (hazard ratio 4.06, 95\% CI 1.55-7.72; $\mathrm{p}=0.0025$ by log-rank test; fig. 2). Multivariate time-to-event analysis confirmed the better BOS-free survival of patients receiving azithromycin, with a hazard ratio of 0.27 compared to placebo after adjustment for covariates (95\% CI 0.092-0.816; $\mathrm{p}=0.020$ by Cox proportional hazards analysis).

Obliterative bronchiolitis $<2$ yrs after transplantation could be assessed histologically in nine patients of the placebo group (seven autopsies and two retransplantations) and in five patients of the azithromycin group (four autopsies and one retransplantation). Autopsy was refused by the patients' relatives in one patient of the placebo group and in two patients of the azithromycin group. Obliterative bronchiolitis was diagnosed in four patients receiving placebo and one receiving azithromycin $(\mathrm{p}=0.36)$, all of whom were nonresponders (i.e. further FEV1 deterioration) to open-label azithromycin treatment.

\section{Survival analysis}

Survival between both groups was similar (fig. 2). There were eight $(18.6 \%)$ deaths in the placebo group, as compared with six $(15.0 \%)$ deaths in the azithromycin group $(\mathrm{p}=0.64$ by log-rank analysis). Multivariate time-to-event analysis showed a hazard ratio of 0.25 for azithromycin compared to placebo after adjustment for covariates $(95 \%$ CI $0.05-1.38$; $\mathrm{p}=0.11$ by Coxproportional hazards analysis, survival not censored for retransplantation or study drug discontinuation). In the case of censoring of survival at retransplantation or study drug discontinuation, additional time-to-event analysis showed a hazard ratio of 0.15 for azithromycin (95\% CI 0.01-2.27; $\mathrm{p}=0.17$ ).

Six deaths were attributed to pneumonia with sepsis (four in the placebo group and two in the azithromycin group) 


\begin{tabular}{|c|c|c|c|}
\hline Donor age at transplantation yrs & $44.0(37.0-54.0)$ & $51.0(36.5-57.5)$ & 0.20 \\
\hline Recipient age at transplantation yrs & $55.1(44.2-59.4)$ & $56.1(47.7-61.2)$ & 0.76 \\
\hline Males/females & $20 / 23(46.5 / 53.5)$ & $17 / 23(42.5 / 57.5)$ & 0.71 \\
\hline Emphysema (COPD) & $24(55.8)$ & $17(42.5)$ & \\
\hline Pulmonary fibrosis & $10(23.3)$ & $9(22.5)$ & \\
\hline Cystic fibrosis & $6(13.9)$ & $8(20.0)$ & \\
\hline$\alpha_{1}$-Antitrypsin deficiency & $2(4.7)$ & $1(2.5)$ & \\
\hline Pulmonary arterial hypertension & $0(0.0)$ & $2(5.0)$ & \\
\hline Asthma & $1(2.3)$ & $1(2.5)$ & \\
\hline SLTX & $10(23.3)$ & $12(30.0)$ & \\
\hline HLTX & $0(0.0)$ & $2(5.0)$ & \\
\hline Donor-recipient CMV mismatch ${ }^{\#}$ & $9(20.9)$ & $9(22.5)$ & 0.86 \\
\hline Ischaemic time $\min$ & $344.0 \pm 108.7$ & $341.4 \pm 86.6$ & 0.88 \\
\hline \multicolumn{4}{|l|}{ PGD score ${ }^{+}$} \\
\hline Mean PGD score T0-T48 & $1.97 \pm 0.76$ & $1.80 \pm 0.82$ & 0.32 \\
\hline Highest PGD score T0-T48 & $2.49 \pm 0.75$ & $2.23 \pm 0.83$ & 0.17 \\
\hline ICU stay after transplantation days & $8.9 \pm 6.7$ & $7.5 \pm 5.3$ & 0.33 \\
\hline Hospitalisation after transplantation days & $30.9 \pm 8.7$ & $34.9 \pm 25.2$ & 0.82 \\
\hline Time from transplantation to start study treatment days & $36.1 \pm 9.4$ & $35.8 \pm 12.0$ & 0.50 \\
\hline Total duration of study treatment days & $498.2 \pm 224.7$ & $565.7 \pm 224.5$ & 0.17 \\
\hline
\end{tabular}

Data are presented as median (interquartile range), $n(\%)$ or mean $\pm S D$, unless otherwise stated. Patients' characteristics for the patients of the placebo-arm $(n=43)$ and of the azithromycin-arm $(n=40)$. Groups were compared using unpaired, two-tailed t-tests, Mann-Whitney tests or Chi-squared tests. COPD: chronic obstructive pulmonary disease; SSLTx: sequential single-lung transplant; SLTx: single-lung transplant; HLTX: heart-lung transplant; CMV: cytomegalovirus; PGD: primary graft dysfunction; ICU: intensive care unit. " : CMV mismatch was defined as a CMV-positive donor and a CMV-negative recipient; ": ischaemic time was calculated from the time of ischaemia registered for the second lung in case of bilateral transplantation, the heart-lung block in case of heart-lung transplantation or for the first lung in case of unilateral transplantation; ${ }^{+}$: PGD was assessed at $0,12,24$ and $48 \mathrm{~h}$ after transplantation, and mean PGD score was calculated for each patient using PGD score at every timepoint during this $48-h$ interval.

(table 2). Three other patients died from sepsis of nonpulmonary origin (one receiving placebo and two receiving azithromycin) and two patients died due to pulmonary malignancy (one in each group). Two patients (one in each group) died because of respiratory failure due to clinically significant chronic rejection without concurrent infection. One patient in the placebo group died because of respiratory failure due to diffuse alveolar damage of unknown origin. Post mortem verification of the cause of death was not possible due to refusal of autopsy in one patient dying from a pulmonary Aspergillus infection (receiving placebo), one patient dying from chronic rejection (receiving azithromycin) and one patient dying from pulmonary malignancy (receiving azithromycin).

\section{Secondary end-points}

Acute rejection (grade $A$ )

Both total number of acute rejection episodes and number of episodes of grade A2 or higher were similar between groups (supplementary table S3). Analysis correcting for CMV mismatch status confirmed that acute rejection rate was not associated with treatment, since, independent of CMV mismatch and compared with patients not having an acute rejection, the odds for being in treatment were OR 1.61 (95\% CI 0.84-3.10; $\mathrm{p}=0.15)$ and OR 0.87 (95\% CI 0.44-1.74; $\mathrm{p}=0.70)$ for those having one and two or more acute rejections, respectively.

\section{Lymphocytic bronchiolitis (grade B)}

Both total number of lymphocytic bronchiolitis episodes and number of episodes of grade B2 or higher were similar between groups (supplementary table S3).

\section{Infection and airway colonisation}

The pneumonia rates for both CMV and non-CMV pneumonitis were similar between groups (supplementary table S3). The prevalence of airway tract colonisation with Pseudomonas aeruginosa was also similar between groups (table S3). 


\section{TABLE 2 Patients' outcome}

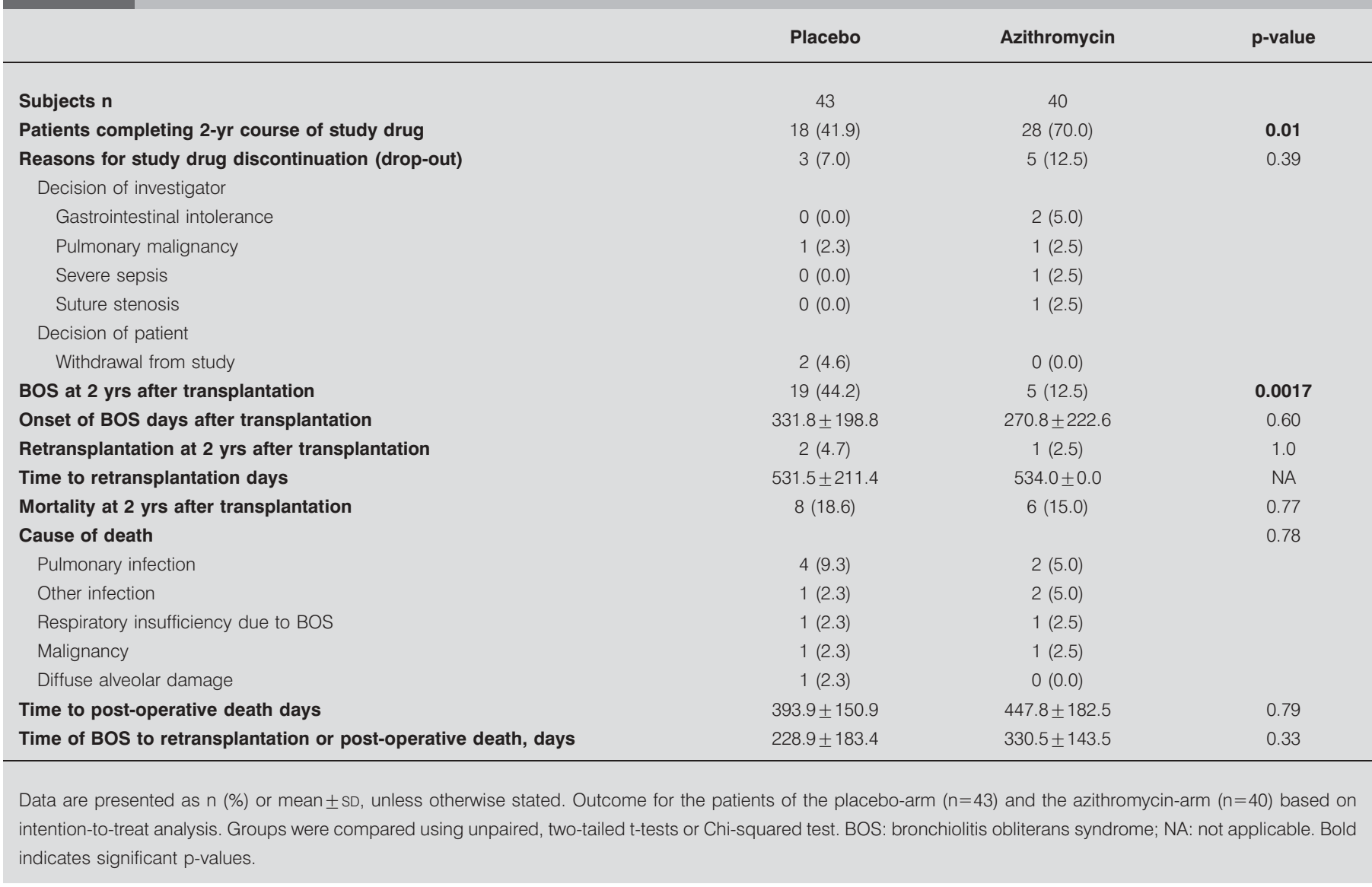

a)

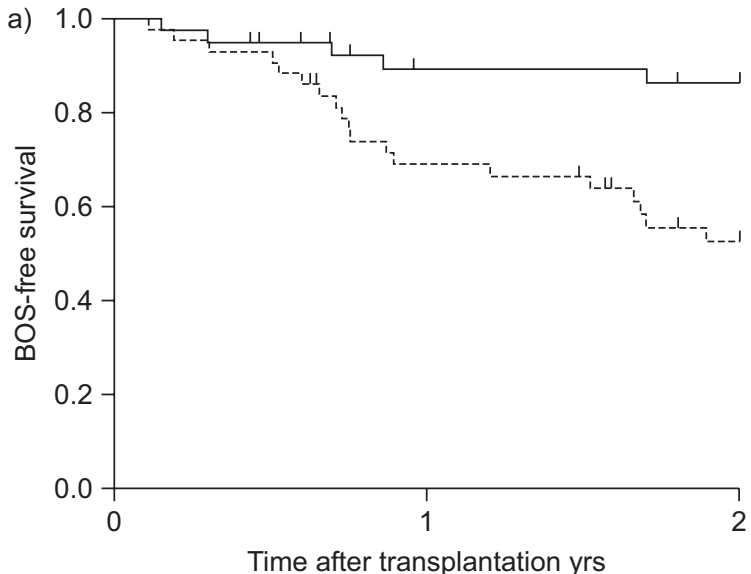

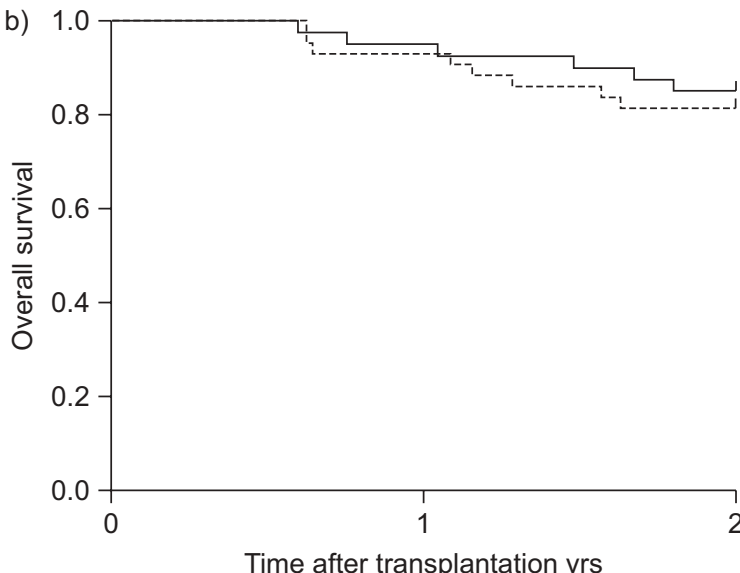

Time after transplantation yrs

At risk $n$

\begin{tabular}{|c|c|c|c|c|c|c|c|c|c|c|}
\hline $\begin{array}{l}\text { At risk } \mathrm{n} \\
\text { Placebo }\end{array}$ & 43 & 41 & 29 & 27 & 18 & $\begin{array}{l}\text { At risk } \mathrm{n} \\
\text { Placebo }\end{array}$ & 43 & 43 & 40 & 38 \\
\hline Azithromycin & 40 & 37 & 31 & 31 & 28 & Azithromycin & 40 & 40 & 38 & 37 \\
\hline
\end{tabular}

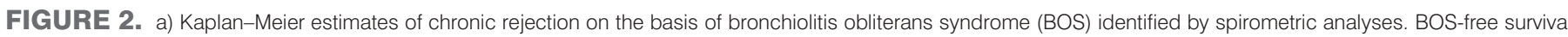

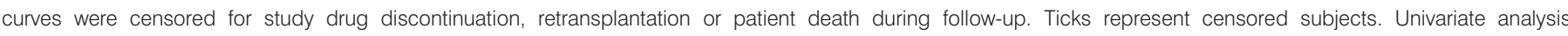

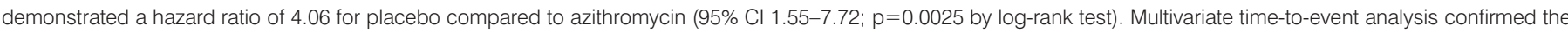

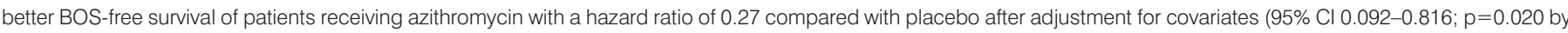

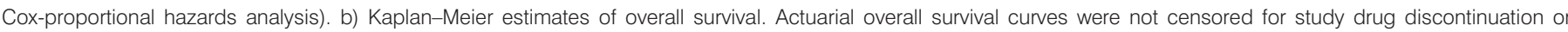

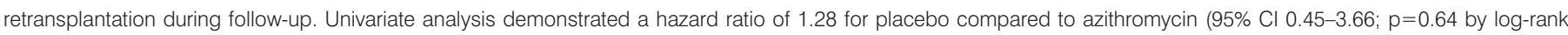

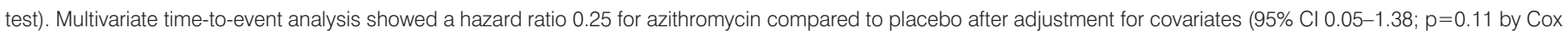
proportional hazards analysis).___ : azithromycin; -----: placebo. 


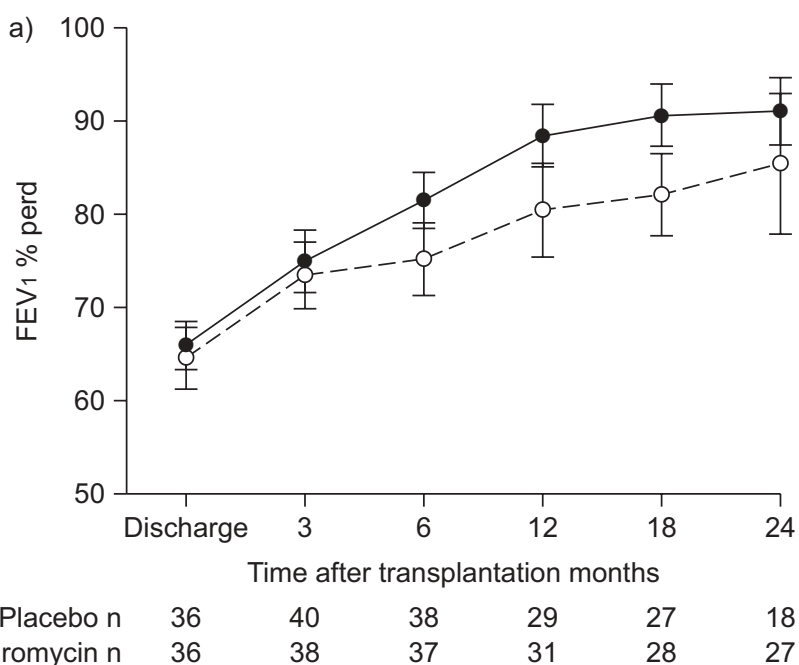

$\begin{array}{rrrrrrr}\text { Placebo n } & 36 & 40 & 38 & 29 & 27 & 18 \\ \text { Azithromycin n } & 36 & 38 & 37 & 31 & 28 & 27\end{array}$

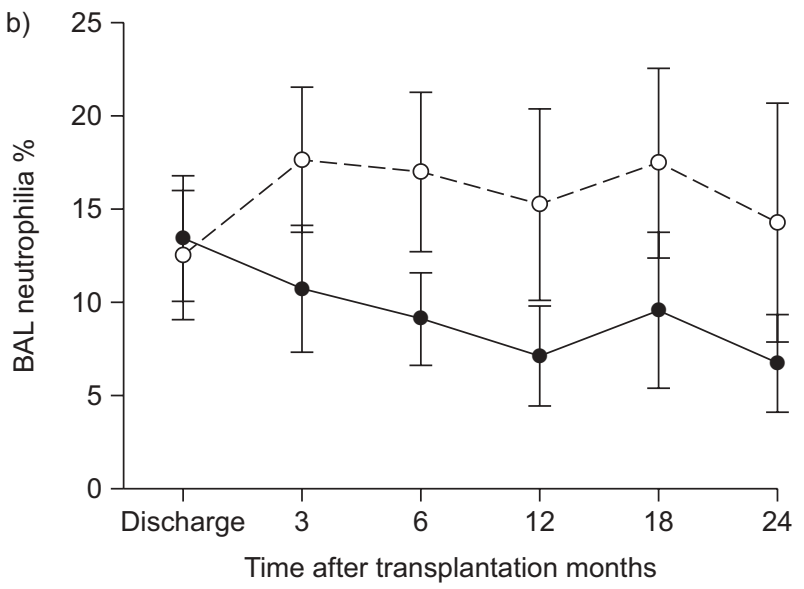

$\begin{array}{rllllll}\text { Placebo n } & 43 & 41 & 38 & 29 & 27 & 18 \\ \text { Azithromycin n } & 37 & 38 & 37 & 31 & 28 & 27\end{array}$

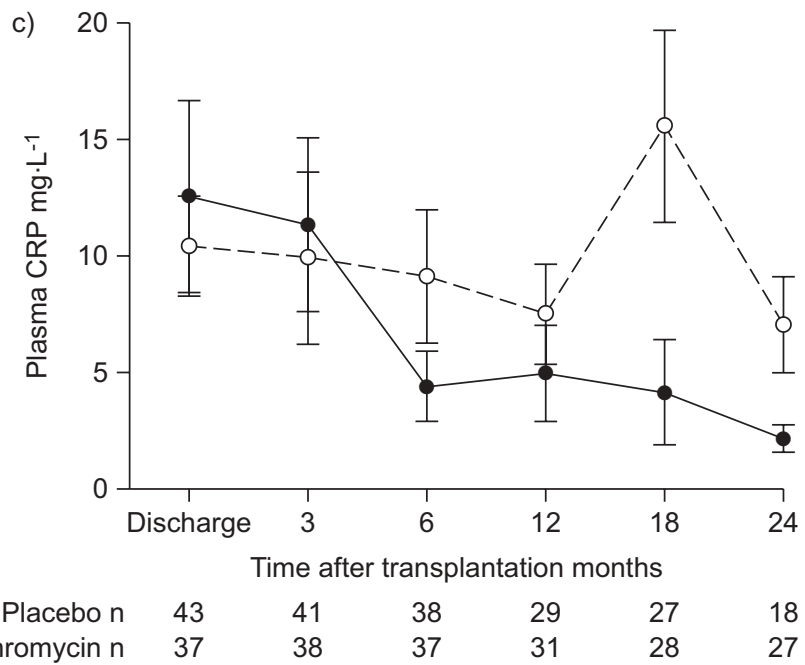

FIGURE 3. Change in a) forced expiratory volume in $1 \mathrm{~s}$ (FEV1) \% predicted $(p=0.028)$, b) bronchoalveolar (BAL) neutrophilia $(p=0.015)$ and c) plasma C-reactive protein (CRP) $(p=0.050)$ over time in both groups assessed at routine follow-up visits in study after transplantation. Data are presented as mean \pm SEM. Mixed models were applied with random intercepts for each subject. The p-values for neutrophilia and CRP were calculated after transformation by Box-Cox to normalise the data. A significant time-by-treatment interaction was observed $(p=0.0011)$ for plasma CRP. ๑: azithromycin; $\bigcirc$ placebo

\section{Gastro-oesophageal reflux}

The majority of patients in each group were assessed by $\mathrm{pH}$ impedance measurement, whereas gastroscopy was performed in only seven patients in each group. Reflux could not be assessed in three patients receiving placebo and in two patients receiving azithromycin due to subjective intolerance of the $\mathrm{pH}$ probe or endoscopic procedure. Time of evaluation after transplantation as well as the prevalence of reflux was not statistically different between groups (supplementary table S3).

\section{Pulmonary function}

FEV1 assessed at scheduled routine follow-up visits was significantly better in patients receiving azithromycin compared to patients receiving placebo $(p=0.028)$ (fig. 3).

\section{Airway and systemic inflammation}

Bronchoalveolar lavage neutrophilia and plasma C-reactive protein assessed at scheduled routine follow-up visits were significantly lower in patients receiving azithromycin compared with patients receiving placebo $(p=0.015$ and $p=0.050$, respectively; fig. 3). A significant time-by-treatment interaction was observed ( $\mathrm{p}=0.0011$ ) for plasma CRP. Mean \pm SEM bronchoalveolar lavage neutrophilia and plasma C-reactive protein levels over time after discharge were $19.4 \pm 3.7$ versus $9.4 \pm 1.9 \%$ $(p=0.028)$ and $14.0 \pm 3.5$ versus $6.7 \pm 1.4 \mathrm{mg} \cdot \mathrm{L}^{-1} \quad(p=0.016)$, respectively, in patients receiving placebo compared to patients receiving azithromycin.

\section{Adverse events}

No allergic reactions or neurologic disorders were seen in either group. Cardiac arrhythmias, all of which were atrial or supraventricular tachyarrythmias, were seen in four $(9.3 \%)$ patients receiving placebo and in one $(2.5 \%)$ patient receiving azithromycin $(\mathrm{p}=0.36)$. No ventricular tachyarrythmias were seen. Gastrointestinal disorders were seen in one patient $(2.3 \%)$ receiving placebo, which had a mild episode of pseudomembranous colitis, and in three $(7.5 \%)$ patients receiving azithromycin $(p=0.35)$. The latter reported nausea and diarrhea after intake of azithromycin, which led to study drug discontinuation in two patients, one of which also had an episode of pseudomembranous colitis.

\section{Open-label azithromycin treatment}

Open-label azithromycin treatment was initiated in 23 patients, 18 receiving placebo and five receiving azithromycin, who developed BOS at a mean \pm SD of $328.1 \pm 199.9$ days after transplantation (supplementary table S4). After initiation of open-label azithromycin, FEV1 significantly improved (to BOS stage 0$)$ in $10(55.6 \%)$ out of 18 patients receiving placebo and in two $(40.0 \%)$ out of five patients receiving azithromycin, who afterwards admitted not taking their study medication properly (supplementary figure S1). No improvement was seen in seven $(38.8 \%)$ patients receiving placebo and three $(60.0 \%)$ patients receiving azithromycin. In one patient receiving placebo change in FEV1 over time could not be assessed due to an episode of severe sepsis within 30 days after initiation, necessitating stopping open-label treatment. Responders generally developed BOS earlier and had a higher bronchoalveolar neutrophilia when BOS was diagnosed compared to nonresponders (table S4). No statistical difference was seen in acute rejection, lymphocytic bronchiolitis or infection rate, or 
in airway colonisation or gastro-oesophageal reflux between responders and nonresponders in either group (supplementary table S4).

\section{DISCUSSION}

Chronic rejection, or its clinical correlate BOS, remains the leading cause of death after lung transplantation despite the use of immunosuppressive therapy $[1,8,9]$. Azithromycin has been shown to improve FEV1 and survival in some lung transplant patients with BOS [16-25]. We hypothesised that prophylactic azithromycin treatment might help prevent chronic rejection and would improve outcomes after lung transplantation. This single-centre, double-blind, placebocontrolled trial of azithromycin given in addition to conventional immunosuppression demonstrated a significant improvement of BOS-free survival 2 yrs after lung transplantation. Overall survival was similar in patients receiving placebo and in patients receiving azithromycin. Secondary end-points, such as the rate of acute rejection, lymphocytic bronchiolitis, pulmonary infection and the prevalence of airway colonisation or gastro-oesophageal reflux were similar between both groups. Yet, patients receiving azithromycin demonstrated better FEV1, as well as significantly lower airway neutrophilia and systemic C-reactive protein levels over time compared to those receiving placebo. The most important symptoms associated with azithromycin treatment were nausea and diarrhoea, reported in $7.5 \%$ of patients receiving azithromycin. Open-label azithromycin improved pulmonary function in half of the patients with established BOS. In the absence of notable differences in acute rejection, lymphocytic bronchiolitis, infection, colonisation and reflux, the positive result in terms of chronic rejection can probably be attributed to the known anti-inflammatory and immunomodulatory properties of azithromycin [22, 30], attenuating local airway and systemic inflammation resulting from these alloimmunologic and nonalloimmunologic events affecting the pulmonary allograft. The comparable survival between groups is most likely due to the initiation of open-label azithromycin treatment in patients with established BOS, possibly resulting in a better outcome in those patients with subsequent improvement of pulmonary function [25]. The observed differences in prevalence of BOS, BOS-free survival, and changes in FEV1, airway and systemic inflammation are substantial, and may even suggest a change in current practice; however, longer follow-up is required to confirm the magnitude and durability of the observed effects. Moreover, some additional considerations should be taken into account when implementing azithromycin as prophylactic therapy. Nevertheless possible adverse events are rare; one should be aware of the most common side-effects of nausea, vomiting, diarrhoea or abdominal pain due to stimulation of gut motility by azithromycin. Another consideration one should take into account in case of long-term prophylaxis is the potential selection of antibiotic-resistant organisms in the normal flora or pathogenic bacteria, the full impact of which may only be evident after several years of treatment. Whether this prophylactic approach is superior for long-term survival to treating BOS once it has occurred can only truly be demonstrated in a larger multicentre study comparing both strategies.

\section{SUPPORT STATEMENT}

R. Vos is a research fellow, and B.M. Vanaudenaerde, D.E. Van Raemdonck and L.J. Dupont senior research fellows of the Research Foundation-Flanders (FWO G.0518.06, G.0643.08, G.0723.10). G.M. Verleden is holder of the GlaxoSmithKline Chair in Respiratory Pharmacology at the Katholieke Universiteit Leuven (Leuven, Belgium).

\section{CLINICAL TRIAL}

This study is registered at ClinicalTrials.gov with clinical trial identifier number NCT01009619 and in the ISRCTN registry with indentifier number 36220396.

\section{STATEMENT OF INTEREST}

A statement of interest for G.M. Verleden can be found at www.erj. ersjournals.com/site/misc/statements.xhtml

\section{ACKNOWLEDGEMENTS}

The authors wish to acknowledge the following persons for their support in the current trial: C. Jans, C. Rosseel and M. Meelberghs (all Lung Transplant Unit Outpatient Clinic, University Hospital Gasthuisberg, Leuven, Belgium); A. Van Den Eeckhout, C. Dooms, J. Foulon and F. Vandeweyer (all Dept of Bronchoscopy, University Hospital Gasthuisberg); F. Rochette and Y. Dewandeleer (both Dept of Pulmonary Function, University Hospital Gasthuisberg); D. Van Raemdonck, W. Coosemans, H. Decaluwé, P. De Leyn, P. Nafteux and T. Lerut (all Dept of Thoracic Surgery, University Hospital Gasthuisberg); L. Hutsebaut, K. De Gieter and T. De Rijdt (Dept of Experimental Pharmacy, University Hospital Gasthuisberg); and G. Mariën (Dept Laboratory Medicine, University Hospital Gasthuisberg)

\section{REFERENCES}

1 Christie JD, Edwards LB, Aurora $\mathrm{P}$, et al. The registry of the international society for heart and lung transplantation: twentysixth official adult lung and heart-lung transplantation report 2009. J Heart Lung Transplant 2009; 28: 1031-1049.

2 Verleden GM, Dupont LJ, Van Raemdonck DE, et al. Lung transplantation: a 15 year single center experience. Clin Transpl 2007: 121-130.

3 Sayegh MH, Carpenter CB. Transplantation 50 years laterprogress, challenges, and promises. N Engl J Med 2004; 351: 2678-2680.

4 Lin HM, Kauffman HM, McBride MA, et al. Center-specific graft and patient survival rates: 1997 United Network for Organ Sharing (UNOS) report. JAMA 1998; 280: 1153-1160.

5 Burke CM, Theodore J, Dawkins KD, et al. Post-transplant obliterative bronchiolitis and other late lung sequelae in human heart-lung transplantation. Chest 1984; 86: 824-829.

6 Estenne M, Hertz MI. Bronchiolitis obliterans after human lung transplantation. Am J Respir Crit Care Med 2002; 166: 440-444.

7 Stewart S, Fishbein MC, Snell GI, et al. Revision of the 1996 working formulation for the standardization of nomenclature in the diagnosis of lung rejection. Heart Lung Transplant 2007; 26: 1229-1242.

8 Verleden GM, Vos R, De Vleeschauwer SI, et al. Obliterative bronchiolitis following lung transplantation: from old to new concepts? Transpl Int 2009; 22: 771-779.

9 Estenne M, Maurer JR, Boehler A, et al. Bronchiolitis obliterans syndrome 2001: an update of the diagnostic criteria. J Heart Lung Transplant 2002; 21: 297-310.

10 Sato M, Keshavjee S. Bronchiolitis obliterans syndrome: alloimmune-dependent and -independent injury with aberrant tissue remodeling. Semin Thorac Cardiovasc Surg 2008; 20: 173-182. 
11 Belperio JA, Weigt SS, Fishbein MC, et al. Chronic lung allograft rejection: mechanisms and therapy. Proc Am Thorac Soc 2009; 6: 108-121.

12 Hopkins PM, McNeil K. Evidence for immunosuppression in lung transplantation. Curr Opin Organ Transplant 2008; 13: 477-483.

13 Snell GI, Westall GP. Immunosuppression for lung transplantation: evidence to date. Drugs 2007; 67: 1531-1539.

14 Scott AI, Sharples LD, Stewart S. Bronchiolitis obliterans syndrome: risk factors and therapeutic strategies. Drugs 2005; 65: 761-771.

15 Borro JM, Bravo C, Solé A, et al. Conversion from cyclosporine to tacrolimus stabilizes the course of lung function in lung transplant recipients with bronchiolitis obliterans syndrome. Transplant Proc 2007; 39: 2416-2419.

16 Gerhardt SG, McDyer JF, Girgis RE, et al. Maintenance azithromycin therapy for bronchiolitis obliterans syndrome: results of a pilot study. Am J Respir Crit Care Med 2003; 168: 121-125.

17 Verleden GM, Dupont LJ. Azithromycin therapy for patients with bronchiolitis obliterans syndrome after lung transplantation. Transplantation 2004; 77: 1465-1467.

18 Yates B, Murphy DM, Forrest IA, et al. Azithromycin reverses airflow obstruction in established bronchiolitis obliterans syndrome. Am J Respir Crit Care Med 2005; 172: 772-775.

19 Shitrit D, Bendayan D, Gidon S, et al. Long-term azithromycin use for treatment of bronchiolitis obliterans syndrome in lung transplant recipients. J Heart Lung Transplant 2005; 24: 1440-1443.

20 Porhownik NR, Batobara W, Kepron W, et al. Effect of maintenance azithromycin on established bronchiolitis obliterans syndrome in lung transplant patients. Can Respir J 2008; 15: 199-202.
21 Gottlieb J, Szangolies J, Koehnlein T, et al. Long-term azithromycin for bronchiolitis obliterans syndrome after lung transplantation. Transplantation 2008; 85: 36-41.

22 Verleden GM, Vanaudenaerde BM, Dupont LJ, et al. Azithromycin reduces airway neutrophilia and interleukin-8 in patients with bronchiolitis obliterans syndrome. Am J Respir Crit Care Med 2006; 174: 566-570.

23 Vanaudenaerde BM, Meyts I, Vos R, et al. A dichotomy in bronchiolitis obliterans syndrome after lung transplantation revealed by azithromycin therapy. Eur Respir J 2008; 32: 832-843.

24 Vos R, Vanaudenaerde BM, Ottevaere A, et al. Long-term azithromycin for bronchiolitis obliterans syndrome: divide and conquer? J Heart Lung Transplant 2010; [Epub ahead of print DOI: 10.1016/j.healun.2010.05.023].

25 Jain R, Hachem RR, Morrell MR, et al. Azithromycin is associated with increased survival in lung transplant recipients with bronchiolitis obliterans syndrome. J Heart Lung Transplant 2010; 29: 531-537.

26 van den Berg JW, van Enckevort PJ, TenVergert EM, et al. Bronchiolitis obliterans syndrome and additional costs of lung transplantation. Chest 2000; 118: 1648-1652.

27 American Thoracic Society. Standardization of spirometry, 1994 update: Am J Respir Crit Care Med 1995; 152: 1107-1136.

28 Morris JF. Spirometry in the evaluation of pulmonary function. West J Med 1976; 125: 110-118.

29 Blondeau K, Mertens V, Vanaudenaerde BA, et al. Gastroesophageal reflux and gastric aspiration in lung transplant patients with or without chronic rejection. Eur Respir J 2008; 31: 707-713.

30 Giamarellos-Bourboulis EJ. Macrolides beyond the conventional antimicrobials: a class of potent immunomodulators. Int $J$ Antimicrob Agents 2008; 31: 12-20. 